International Journal of Pure and Applied Mathematics

Volume 105 No. 4 2015, 549-560

ISSN: 1311-8080 (printed version); ISSN: 1314-3395 (on-line version)

url: http://www.ijpam.eu

doi: http://dx.doi.org/10.12732/ijpam.v105i4.1

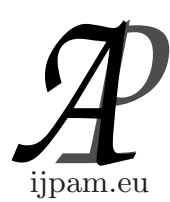

\title{
NUMERICAL TREATMENT OF FREDHOLM INTEGRAL EQUATIONS OVER $(0,+\infty)$ USING HILBERT TRANSFORM
}

\author{
F. Samadi ${ }^{1}$, A. Omidi ${ }^{2}$, R. Mohammad Hoseini ${ }^{3} \S$ \\ ${ }^{1}$ Department of Mathematics \\ Payame Noor University (PNU) \\ P.O. Box 19395-3697, Tehran, IRAN \\ ${ }^{2}$ Department of Applied Mathematics \\ Razi University \\ Kermanshah, IRAN \\ ${ }^{3}$ Department of Mathematics \\ Faculty of Science \\ Islamic Azad University \\ Central Tehran Branch \\ Tehran, IRAN
}

\begin{abstract}
In this paper, we present two types of integral equations of the second kind over semiaxis. Then we introduce a new numerical method by using the product rule, Nyström method and Hilbert Transform and prove that the proposed procedures are stable and convergent. The method reduces the integral equation to a system of linear algebraic equations. Some numerical examples are presented to show the efficiency and accuracy of the method.
\end{abstract}

AMS Subject Classification: 45B05, 45G10

Key Words: Fredholm integral equations, Nyström interpolation, Hilbert transform, Gaussian quadrature rules

Received: January 29, 2015

(C) 2015 Academic Publications, Ltd. url: www.acadpubl.eu

${ }^{\S}$ Correspondence author 


\section{Introduction}

Many problems in engineering and mechanics give rise to the second-kind twodimensional integral equations. For example, it is usually required to solve Fredholm integral equations in the calculation of plasma physics [1]. McKee et al. [2] have shown that a class of nonlinear telegraph equations is equivalent to two-dimensional Volterra integral equations.

Consider the Fredholm integral equation (FIE) of the second kind

$$
f(y)-\int_{0}^{+\infty} k(x, y) f(x) u(x) d x=g(y), \quad y \in(0,+\infty)
$$

where $k(x, y)$ and $g(y)$ are given functions and $u(x)=x^{\gamma} e^{\frac{-x}{2}}, \gamma \geq 0$, is a generalized Laguerre weight, and $f$ is the unknown function and should be determined. In this paper we propose a new numerical method, based on the polynomial approximation, for computing the solution of $(1)$. Where $y \in(-1,1)$, $u(x)$ is a Jacobi weight and the integral is defined on interval $(-1,1)$. There has been much work on developing and analyzing the numerical methods for solving one-dimensional Fredholm integral equations (see, for example, [3, 4, 5, 6] and the references cited therein). But the analysis of computational methods for FIEs on the real semiaxis seem to have been discussed in only a few papers. In our case the integral is defined on the real semiaxis and includes a nonclassical weight. for a long time the theory of the weighted polynomial approximation on the real semiaxis has been neglected. Some theorems that are useful to the aims of this paper have only recently been proved $[7,8]$.

In the present work, we apply the product rule, Nyström method and Hilbert Transform to solve a class of nonlinear of the second kind on the real semiaxis. Our method reduces this problem into a linear system of algebraic equations. To solve the obtained linear system, an iterative method is provided. We prove that, under some conditions, the sequence of approximate solutions produced by the iterative method converges to the exact solution of the linear system.

\section{Notations and Preliminaries}

In the sequel $C$ will denote any positive constant which can be different in different formulas. Moreover, $C \neq C(a, b, \ldots)$ will be used to mean the that constant $C$ is independent of $a, b, \ldots$ The notation $A \sim B$, where $A$ and $B$ are 
positive quantities depending on some parameters, will be used if and only if $\left(\frac{A}{B}\right)^{ \pm 1} \leq C$, with $C$ positive constant independent of the above parameters.

Throughout the paper $\theta$ will denote a fixed real number with $0<\theta<1$, which can be different in different formulas. Denoted by $a_{m}=a_{m}(u)$ the Maskhar-Rachmanov-Saff number (in the sequel we say shortly M-R-S number). It was proved that (see [11])

$$
a_{m}=a_{m}(u)=\Gamma m \sim m .
$$

\subsection{Functional Spaces}

Let $u(x)=x^{\gamma} e^{\frac{-x}{2}}, x, \gamma \geq 0$ and we define the space

$$
C_{u}=\left\{f \in C((0, \infty)): \lim _{\substack{x \rightarrow \infty \\ x \rightarrow 0}}(f u)(x)=0\right\},
$$

where $C(J)$ denotes the collection of all continuous functions on $J \subseteq[0, \infty)$.

If $\gamma=0$, the space $C_{u}$ consists of all continuous functions on $[0, \infty)$ such that $\lim _{x \rightarrow \infty}(f u)(x)=0$. This space equipped with the following norm

$$
\|f\|_{C_{u}}=\|f u\|_{\infty}=\sup _{x \geq 0}|(f u)(x)|
$$

is a Banach space.

In order to introduce a subspace of $C_{u}$ we proceed as in [9] defining the following main part of the modulus of smoothness

$$
\Omega_{\varphi}^{r}(f, \tau)_{u}=\sup _{0<h \leq \tau}\left\|\left(\Delta_{h \varphi}^{r} f\right) u\right\|_{I_{r h}},
$$

where $r=1,2, \ldots, \varphi(x)=\sqrt{x}$ and $\|\cdot\|_{I_{r h}}$ denotes the uniform norm on the interval $I_{r h}=\left[8 r^{2} h^{2}, C h^{*}\right]$, where $h^{*}=h^{-2}, C$ is a fixed constant and

$$
\Delta_{h \varphi}^{r} f(x)=\sum_{i=0}^{r}(-1)^{i}\left(\begin{array}{l}
r \\
i
\end{array}\right) f\left(x+\left(\frac{r}{2}-i\right) h \varphi(x)\right) .
$$

We recall that the introduced modulus $\Omega_{\varphi}^{r}(f, \tau)_{u}$, can be estimated by means of the following estimate

$$
\Omega_{\varphi}^{r}(f, \tau)_{u} \leq C \sup _{0<h \leq \tau} h^{r}\left\|f^{(r)} \varphi^{r} u\right\|_{r h}, \quad C \neq C(f, \tau)
$$

if the norm at the right-hand side is bounded. 
Now let $\mathcal{P}_{m}$ be the set of all algebraic polynomials of degree at most $m$ and we denote by

$$
E_{m}(f)_{u}=\inf _{P \in \mathcal{P}_{m}}\|(f-P) u\|_{\infty}
$$

the error of best approximation of $f \in C_{u}$. It is well known that for all $f \in C_{u}$ the following weak Jackson and Salem-Stechkin inequalities hold

$$
\begin{gathered}
E_{m}(f)_{u} \leq C \int_{0}^{\frac{\sqrt{a_{m}}}{m}} \frac{\Omega_{\varphi}^{r}(f, t)_{u}}{t} d t \\
\Omega_{\varphi}^{r}\left(f, \frac{\sqrt{a_{m}}}{m}\right)_{u} \leq C\left(\frac{\sqrt{a_{m}}}{m}\right)^{r} \sum_{k=0}^{m}\left(\frac{k+1}{\sqrt{a_{k}}}\right)^{r} \frac{E_{k}(f)_{u}}{k+1}
\end{gathered}
$$

where in both cases $r<m$ and $C \neq C(m, f)$, (see[13]).

For differentiable functions we introduce the Sobolev-type spaces

$$
W_{s}=W_{s}(u)=\left\{f \in C_{u}: f^{(s-1)} \in A C\left(\mathbb{R}^{+}\right) \text {and }\left\|f^{(s)} \varphi^{s} u\right\|_{\infty}<+\infty\right\},
$$

where $s=1,2, \ldots$ and $A C\left(\mathbb{R}^{+}\right)$is the set of all absolutely continuous functions in $\mathbb{R}^{+}$. In $W_{s}$ we introduce the norm

$$
\|f\|_{W_{s}}=\|f u\|_{\infty}+\left\|f^{(s)} \varphi^{s} u\right\|_{\infty} .
$$

It is useful to observe that, for every $f \in W_{s}$, by (5) and

$$
\Omega_{\varphi}^{s}(f, t)_{u} \leq C\left\|f^{(s)} \varphi^{s} u\right\|_{\infty} t^{s}
$$

(see [9]), we deduce that

$$
E_{m}(f)_{u} \leq C\left(\frac{\sqrt{a_{m}}}{m}\right)^{s}\|f\|_{W_{s}}, \quad m>s .
$$

\subsection{Lagrange Interpolation}

Let $w(x)=x^{\alpha} e^{-x}, x>0, \alpha>-1$, be a generalized Laguerre weight and let $\left\{p_{m}(w)\right\}_{m \in \mathbb{N}}$ be the sequence of the Laguerre orthonormal polynomials with positive leading coefficient, i.e.

$$
\begin{gathered}
p_{m}(w)=\gamma_{m}(w) x^{m}+\cdots, \quad \gamma_{m}(w)>0 \\
\int_{\mathbb{R}^{+}} p_{m}(w, x) p_{n}(w, x) w(x) d x=\delta_{m, n}
\end{gathered}
$$


The zeros $x_{k}:=x_{m, k}(w), k=1, \ldots, m$, of $p_{m}(w)$ satisfy [10]

$$
\frac{C}{m}<x_{1}<\ldots<x_{m}=4 m+2 \alpha+2-C(4 m)^{\frac{1}{3}} .
$$

We define the integer $j=j(m)$ by

$$
x_{j}=\min _{1 \leq k \leq m}\left\{x_{k}: x_{k} \geq \theta a_{m}\right\}
$$

with $m$ sufficiently ( say $m>m 0$ ) Let us introduce a Lagrange polynomial $L_{m+1}^{*}(w, f)$ which interpolates $f \in C\left(\mathbb{R}^{+}\right)$on the $m+1$ points $x_{1}, x_{2}, \ldots, x_{m}, a_{m}$, i.e.

$$
\begin{aligned}
& L_{m+1}^{*}(w, f ; x)=\sum_{k=1}^{m} l_{k}(x) \frac{a_{m}-x}{a_{m}-x_{k}} f\left(x_{k}\right)+\frac{p_{m}(w, x)}{p_{m}\left(w, a_{m}\right)} f\left(a_{m}\right) \\
&=\sum_{k=1}^{m+1} l_{k}^{*}(x) f\left(x_{k}\right) \\
& l_{k}^{*}(x)=l_{k}(x) \frac{a_{m}-x}{a_{m}-x_{k}}, k=1, \ldots, m, \quad l_{m+1}^{*}(x)=\frac{p_{m}(w, x)}{p_{m}\left(w, a_{m}\right)}
\end{aligned}
$$

where $l_{k}(x), k=1, \ldots, m$ are the fundamental Lagrange polynomials based on the zeros of $p_{m}(w)$. Now, for $m$ sufficiently large (say $m>m_{0}$ ), let $\tilde{\mathcal{P}}_{m}$ be the collection of polynomials of $\mathcal{P}_{m}$ having $x_{j}(w), \ldots, a_{n}(w)$ as zeros, obviously, $\tilde{\mathcal{P}}_{m} \subset \mathcal{P}_{m}$.

Then for $f \in C_{u}$ we consider the truncated interpolation process (see [10] and [12])

$$
L_{m+1}^{* *}(w, f ; x)=\sum_{k=1}^{j} l_{k}(x) \frac{a_{m}-x}{a_{m}-x_{k}} f\left(x_{k}\right)=\sum_{k=1}^{j} l_{k}^{*}(x) f\left(x_{k}\right) \in \tilde{\mathcal{P}}_{m} .
$$

\subsection{Product Rule}

Now we consider quadrature rule based on the previous interpolation process. A Gaussian-type formula is the so called "product rule",

$$
\int_{0}^{+\infty} f(x) k(x, y) u(x) d x=\sum_{k=1}^{j} f\left(x_{k}\right) \lambda_{k}(y)+e_{m}^{*}(f, y)=: I_{m}(f, y)+e_{m}^{*}(f, y)
$$

where $k:[0,+\infty) \times[0,+\infty) \rightarrow \mathbb{R}, \lambda_{k}(y)=\int_{0}^{+\infty} l_{k}^{*}(x) k(x, y) u(x) d x$, and $e_{m}^{*}(f)$ is the error of the quadrature rule [15]. 


\section{Hilbert Transform}

The Hilbert transform of $f$ are defined as follows [10]

$$
H(f, t)=\int_{0}^{\infty} \frac{f(x)}{x-t} d x, \quad t>0 .
$$

We approximate $H(u f, t)$ by $H_{m}(f, t)$. Also $H_{m}(f, t)$ can be written as

$$
H_{m}(f, t)=\sum_{k=1}^{j} \frac{f\left(x_{k}\right)}{\left(4 m-x_{k}\right)} A_{k}(t)
$$

where

$$
A_{k}(t):=\int_{0}^{\infty}(4 m-x) l_{k}(x) \frac{u(x)}{x-t} d x
$$

with $x_{k}:=x_{m, k}(w)$ and

$$
l_{k}(x):=\frac{p_{m}(x)}{p_{m}^{\prime}\left(x_{k}\right)\left(x-x_{k}\right)}
$$

\section{Numerical Results}

Now we return to the equation

$$
f(y)-\int_{0}^{+\infty} k(x, y) f(x) u(x) d x=g(y), \quad y \in(0,+\infty),
$$

we rewrite this equation in the operator form

$$
(I-\mathcal{K}) f=g
$$

where $I$ is the identity operator. By multiplying both sides of the equation by the weight $u(y)$, we have

$$
(f u)(y)-u(y) \int_{0}^{+\infty} k(x, y) f(x) u(x) d x=(g u)(y), \quad y \in[0,+\infty) .
$$

Now, by using the Hilbert Transform the equation (11) reduced to

$$
\left(\sum_{s=1}^{j} \frac{f\left(x_{s}\right)}{4 m-x_{s}} A_{s}(y)\right)-u(y) \int_{0}^{+\infty} k(x, y)\left(\sum_{s=1}^{j} \frac{f\left(x_{s}\right)}{4 m-x_{s}} A_{s}(x)\right) d x=(g u)(y)
$$


where

$$
A_{s}(t)=\int_{0}^{+\infty}(4 m-x) l_{s}(x) \frac{u(x)}{x-t} d x
$$

Letting $\tilde{f}(y)=\sum_{s=1}^{j} \frac{f\left(x_{s}\right)}{4 m-x_{s}} A_{s}(y)$, and approximating the integral by the product rule, we get

$$
\tilde{f}(y)-u(y) \sum_{k=1}^{j} \tilde{f}\left(x_{k}\right) \frac{\lambda_{k}(y)}{u(x)}=(g u)(y)
$$

where

$$
\lambda_{k}(y)=\int_{0}^{+\infty} l_{k}^{*}(x) k(x, y) u(x) d x .
$$

Now, we apply the Nyström method, and get

$$
\tilde{f}\left(x_{i}\right)-u\left(x_{i}\right) \sum_{k=1}^{j} \tilde{f}\left(x_{k}\right) \frac{\lambda_{k}\left(x_{i}\right)}{u\left(x_{k}\right)}=(g u)\left(x_{i}\right), \quad i=1, \ldots, j,
$$

from (12), we obtain $\tilde{f}\left(x_{k}\right)$, for $k=1, \ldots, j$. Then the approximate solution obtained by $\bar{f}_{m}(y)=\sum_{i=1}^{j} \varphi_{i}(y) \alpha_{i} \in \tilde{\mathcal{P}}_{m}$ with $\varphi_{i}(z)=\frac{l_{i}^{*}(z)}{u\left(x_{i}\right)}$ and $\alpha_{i}=\tilde{f} u\left(x_{i}\right)$.

Theorem 1. We assume that the parameters of the weights $w$ and $u$ satisfy the conditions

$$
\max \left\{0, \frac{\alpha}{2}+\frac{1}{4}\right\} \leq \gamma<\frac{\alpha+1}{2},
$$

Concerning the kernel $k(x, y)=k_{x}(y)=k_{y}(x)$ and the known term $g$, we assume that

$$
\sup _{x \geq 0} u(x)\left\|k_{x}\right\|_{W_{s}}<+\infty, \quad \sup _{y \geq 0} u(x)\left\|k_{y}\right\|_{W_{s}}<+\infty, \quad g \in W_{s}, \quad s \geq 1 \text { integer },
$$

and $K$ is a compact operator. Then we have

$$
\left\|\left(f^{*}-\bar{f}_{m}\right) u\right\| \leq C\left(\frac{\sqrt{a_{m}}}{m \sqrt{m}}\right)^{s}\|f\|_{W_{s}}\left\|k_{y}\right\|_{W_{s}},
$$

where $f^{*}$ is the solution of (10) and $C$ is independent of $m, f^{*}$ and $\bar{f}_{m}$. 
Proof. From $\int_{0}^{+\infty} \frac{w(x)}{u^{2}(x)} d x<+\infty$ and From Proposition 2.3 from [11] we have

$$
u(y)\left|\left(f^{*}-\bar{f}_{m}\right)\right| \leq\left[u(y) E_{M}\left(k_{y} f^{*}\right)_{u^{2}}+e^{-A n}\left\|k_{y} f^{*} u^{2}\right\|\right] .
$$

We denote by $P_{M}$ the polynomial of best approximation of $k_{y} f$ in $C_{u}$, by $Q \in \mathcal{P}_{\left\lfloor\frac{M}{2}\right\rfloor}$ the polynomial of best approximation of $k_{y}$ and by $V \in \mathcal{P}_{\left\lfloor\frac{M}{2}\right\rfloor}$ the polynomial of best approximation of $f^{*}$. Therefore

$$
\begin{aligned}
E_{M}\left(k_{y} f^{*}\right)_{u^{2}} & =\left\|\left(k_{y} f^{*}-p_{M}\right) u^{2}\right\| \leq\left\|\left(f^{*} u\right)\left(k_{y}-Q\right) u\right\|+\left\|(Q u)\left(f^{*}-V\right) u\right\| \\
& \leq\left\|f^{*} u\right\| E_{\left\lfloor\frac{M}{2}\right\rfloor}\left(k_{y}\right)_{u}+\left\|k_{y} u\right\| E_{\left\lfloor\frac{M}{2}\right\rfloor}\left(k_{y}\right)_{u} \\
& \leq C\left(\frac{\sqrt{a_{m}}}{m \sqrt{m}}\right)^{s}\|f\|_{W_{s}}\left\|k_{y}\right\|_{W_{s}},
\end{aligned}
$$

so

$$
\begin{aligned}
u(y)\left|f^{*}-\bar{f}_{m}\right| \leq & \left\|f^{*} u\right\| E_{\left\lfloor\frac{M}{2}\right\rfloor}\left(k_{y}\right)_{u}+\left\|k_{y} u\right\| E_{\left\lfloor\frac{M}{2}\right\rfloor}\left(k_{y}\right)_{u}+e^{-A n}\left\|k_{y} u\right\|\left\|f^{*} u\right\| \\
\leq & \left\|C \frac{\|f\|_{W_{s}}}{(\sqrt{m})^{s}} \log ^{2} m+H_{m}(f, t)\right\| E_{\left\lfloor\frac{M}{2}\right\rfloor}\left(k_{y}\right)_{u}+\left\|k_{y} u\right\| E_{\left\lfloor\frac{M}{2}\right\rfloor}\left(k_{y}\right)_{u} \\
& +C e^{-A n}\left\|k_{y} u\right\|\left\|C \frac{\|f\|_{W_{s}}}{(\sqrt{m})^{s}} \log ^{2} m+H_{m}(f, t)\right\| \\
\leq & C\left(\frac{\sqrt{a_{m}}}{m \sqrt{m}}\right)^{s}\|f\|_{W_{s}}\left\|k_{y}\right\|_{W_{s}} .
\end{aligned}
$$

Consequently

$$
\left\|\left(f^{*}-\bar{f}_{m}\right) u\right\| \leq C\left(\frac{\sqrt{a_{m}}}{m \sqrt{m}}\right)^{s}\|f\|_{W_{s}}\left\|k_{y}\right\|_{W_{s}} .
$$

Here we consider the integral equation of the form

$$
f(y)-\int_{0}^{+\infty} k(x, y) f(x) w(x) d x=g(y), \quad y \in(0,+\infty)
$$

where $w(x)=x^{\alpha} e^{-x}, \alpha>-1$ and $u(x)=\sqrt{w(x)}\left(\frac{x}{1+x}\right)^{a}(1+x)^{b}$. Moreover, let the real constants $a, b$ satisfy the conditions $a>-1+\max \left(\frac{-\alpha}{2}, \frac{1}{4}\right), b<\frac{-1}{4}$. We now offer a way to solve the equation (13). First, we introduce the following product rule

$$
\int_{0}^{+\infty} k(x, y) f(x) w(x) d x=\sum_{i=1}^{m} \lambda_{i}(y) f\left(x_{i}\right)+e_{m}^{*}(f, y)
$$


with

$$
\lambda_{i}(y)=\int_{0}^{+\infty} k(x, y) l_{i}(x) w(x) d x .
$$

Now, we are going to represent a method which is based upon product rule, Nyström method and Hilbert transform for solving this kind of integral equation. For this, we multiply both sides of equation (13) by $u(y)$,

$$
(f u)(y)-u(y) \int_{0}^{+\infty} k(x, y) f(x) u(x) d x=(g u)(y), \quad y \in[0,+\infty)
$$

Now, by using the Hilbert transform the equation (14) reduced to

$$
\left(\sum_{s=1}^{j} \frac{f\left(x_{s}\right)}{4 m-x_{s}} A_{s}(y)\right)-u(y) \int_{0}^{+\infty} k(x, y)\left(\sum_{s=1}^{j} \frac{f\left(x_{s}\right)}{4 m-x_{s}} A_{s}(x)\right) \frac{w(x)}{u(x)} d x=(g u)(y)
$$

where

$$
A_{s}(t)=\int_{0}^{+\infty}(4 m-x) l_{s}(x) \frac{u(x)}{x-t} d x .
$$

Letting $\tilde{f}(y)=\sum_{s=1}^{j} \frac{f\left(x_{s}\right)}{4 m-x_{s}} A_{s}(y)$, and approximating the integral by product rule, we get

$$
\tilde{f}(y)-u(y) \sum_{k=1}^{j} \tilde{f}\left(x_{k}\right) \frac{\lambda_{k}(y)}{u(x)}=(g u)(y)
$$

where

$$
\lambda_{k}(y)=\int_{0}^{+\infty} k(x, y) l_{k}(x) w(x) d x .
$$

Now, we apply the Nyström method, and get

$$
\tilde{f}\left(x_{i}\right)-u\left(x_{i}\right) \sum_{k=1}^{j} \tilde{f}\left(x_{k}\right) \frac{\lambda_{k}\left(x_{i}\right)}{u\left(x_{k}\right)}=(g u)\left(x_{i}\right), \quad i=1, \ldots, j .
$$

By solving this system we can find, $\alpha_{i}, i=1, \ldots, j$ and the approximate solution of integral equation is obtained. 


\section{Numerical Examples}

Example. 1 Let

$$
f(y)-\int_{0}^{+\infty}\left(2 x+5 y^{2}\right) f(x) x^{3} e^{-x} d x=e^{-y}-\frac{3}{8}\left(4+5 y^{2}\right) y .
$$

Here $k(x, y)=\left(2 x+5 y^{2}\right)$. Taking $u(x)=x^{0.26}(1+x)^{-7.26} e^{\frac{-x}{2}}$ and $\theta=0.5$, we can construct and solve the system (15) and in Table. 1, we report the values of the weighted error $u(y)\left|e^{-y}-\bar{f}_{n}(y)\right|$ at some points.

Example. 2 Consider the equation

$$
f(y)-\int_{0}^{+\infty}\left(2 x+5 y^{2}\right) f(x) x^{3} e^{-x} d x=6+\sin (y)
$$

Let $k(x, y)=4\left(x^{2}+y^{3}\right)$ and $u(x)=x^{0.3}(1+x)^{-10.3} e^{\frac{-x}{2}}$ and $\theta=0.5$ and consider the equation in $C_{u}$. In this case the exact solution is $f(x)=\operatorname{Sin}(y)$. We can construct and solve the system (15) and in Table.2, we report the values of the weighted error $u(y)\left|\operatorname{Sin}(y)-\bar{f}_{n}(y)\right|$ at some points.

\begin{tabular}{|l|l|l|l|l|l|}
\hline$n$ & $y=1$ & $y=2$ & $y=3$ & $y=4$ & $y=5$ \\
\hline 8 & $1.38304(-4)$ & $1.23603(-6)$ & $1.61907(-8)$ & $3.67283(-9)$ & $1.85561(-10)$ \\
16 & $1.37651(-4)$ & $1.23711(-5)$ & $1.62284(-8)$ & $3.67433(-9)$ & $1.83498(-10)$ \\
32 & $1.23886(-3)$ & $3.09277(-5)$ & $7.9519(-7)$ & $2.97621(-7)$ & $2.22033(-8)$ \\
64 & $1.37651(-4)$ & $1.23711(-5)$ & $1.62284(-8)$ & $3.67433(-9)$ & $1.83498(-10)$ \\
\hline
\end{tabular}

Table 1: Weighted errors $\left|\left(e^{-y}-\bar{f}_{n}(y)\right) u(y)\right|$ at the points $y=$ $1,2,3,4,5$.

\begin{tabular}{|l|l|l|l|l|l|}
\hline$n$ & $y=1$ & $y=2$ & $y=3$ & $y=4$ & $y=5$ \\
\hline 8 & $5.01121(-6)$ & $9.81198(-9)$ & $4.70841(-11)$ & $5.01489(-12)$ & $1.37181(-13)$ \\
16 & $5.01616(-6)$ & $9.80891(-9)$ & $4.70222(-11)$ & $5.01655(-12)$ & $1.37341(-13)$ \\
32 & $5.01616(-6)$ & $9.80891(-9)$ & $4.70222(-11)$ & $5.01655(-12)$ & $1.37341(-13)$ \\
64 & $5.01616(-6)$ & $9.80891(-9)$ & $4.70222(-11)$ & $5.01655(-12)$ & $1.37341(-13)$ \\
\hline
\end{tabular}

Table 2: Weighted errors $\left|\left(\operatorname{Sin}(y)-\bar{f}_{n}(y)\right) u(y)\right|$ at the points $y=$ $1,2,3,4,5$. 


\section{References}

[1] Farengo R, Lee YC, Guzdar PN. An electromagnetic integral equation: application to microtearing modes. Phys Fluids 1983;26:3515-23.

[2] McKee S, Tang T, Diogo T. An Euler-type method for two-dimensional Volterra integral equations of the first kind. IMA J Numer Anal 2000;20:423-40.

[3] Atkinson KE. A survey of numerical methods for the solution of Fredholm integral equations of the second kind. Philadelphia: SIAM; 1976.

[4] Atkinson KE. The numerical solution of integral equations of the second kind. Cambridge: Cambridge University Press; 2011.

[5] Baker CT. The numerical treatment of integral equations. Oxford: Clarendon Press; 1978.

[6] Delves LM, Mohamed JL. Computational methods for integral equations. Cambridge: Cambridge University Press; 2011.

[7] DE Bonis, M. C., MAStroianni, G. \& VigGiano, M. (2002) Kfunctionals, moduli of smoothness and weighted best approximation on the semiaxis. Functions, Series, Operators (L. Leindler, F. Schipp \& J. Szabados eds). Budapest: Janos Bolyai Mathematical Society, pp. 181211.

[8] MAStroiAnNi, G. \& SZABADOS, J. (2007) Polynomial approximation on the real semiaxis with generalized Laguerre weights. Stud. Univ. BabesBolyai Math., 52, 105-128.

[9] G. Mastroianni and J. Szabados, Polynomial approximation on the real semiaxis with generalized Leguerre weights, Std. Univ. Babes-Bolyai Math. 52 (4) (2007) 105-128.

[10] M.C. De Bonis and B. Della Vecchia and G. Mastroianni, Approximation of the Hilbert Transform on the real semiaxis using Lagurre zeros, Journal of Computational and Applied Mathematics 140 (2002) 209-229.

[11] D. Occorsio, Extended Lagrange interpolation in weighted uniform norm, Applied Numerical Mathematics 211 (2009) 10-22. 
[12] C. Laurita and G. Mastroianni, Projection method for Fredholm integral equations of the real semiaxis, J. Integral Equations Appl., 21(4) (2009) $559-596$.

[13] Luisa Fermo, A Nyström method for a class of Fredholm integral equations of the third kind on unbounded domains, Applied Numerical Mathematics 59 (2009) 2970-2989.

[14] G. Mastroianni and G.V. Milovanovic, Some numerical methods for secondkind Fredholm integral equations on the real semiaxis, IMA Journal of Numerical Analysis 29 (2009) 1046-1066.

[15] G. Mastroianni and D. Occorsio, Some quadrature formulae with nonstandard weights, SIAM Journal on Numerical Analysis 235 (2010) 602-614.

[16] G. Mastroianni and G. Monegato, Truncated quadrature rules over $(0,+\infty)$ and Nyström-type methods, SIAM J. Numer. Anal. 41 (2003) 1870-1892. 